\title{
Lagrange form of the nonlinear Schrödinger equation for low-vorticity waves in deep water
}

\author{
Anatoly Abrashkin ${ }^{1}$ and Efim Pelinovsky ${ }^{2,3}$ \\ ${ }^{1}$ National Research University Higher School of Economics (HSE), 25/12 Bolshaya Pecherskaya Str., \\ 603155 Nizhny Novgorod, Russia \\ ${ }^{2}$ Institute of Applied Physics RAS, 46 Ulyanov Str., 603950 Nizhny Novgorod, Russia \\ ${ }^{3}$ Nizhny Novgorod State Technical University n.a. R. Alekseev, 24 Minina Str., 603950 Nizhny Novgorod, Russia \\ Correspondence to: Efim Pelinovsky (pelinovsky@hydro.appl.sci-nnov.ru)
}

Received: 28 November 2016 - Discussion started: 14 December 2016

Revised: 18 April 2017 - Accepted: 21 April 2017 - Published: 6 June 2017

\begin{abstract}
The nonlinear Schrödinger (NLS) equation describing the propagation of weakly rotational wave packets in an infinitely deep fluid in Lagrangian coordinates has been derived. The vorticity is assumed to be an arbitrary function of Lagrangian coordinates and quadratic in the small parameter proportional to the wave steepness. The vorticity effects manifest themselves in a shift of the wave number in the carrier wave and in variation in the coefficient multiplying the nonlinear term. In the case of vorticity dependence on the vertical Lagrangian coordinate only (Gouyon waves), the shift of the wave number and the respective coefficient are constant. When the vorticity is dependent on both Lagrangian coordinates, the shift of the wave number is horizontally inhomogeneous. There are special cases (e.g., Gerstner waves) in which the vorticity is proportional to the squared wave amplitude and nonlinearity disappears, thus making the equations for wave packet dynamics linear. It is shown that the NLS solution for weakly rotational waves in the Eulerian variables may be obtained from the Lagrangian solution by simply changing the horizontal coordinates.
\end{abstract}

\section{Introduction}

The nonlinear Schrödinger (NLS) equation was first derived by Zakharov in 1967 (English edition; Zakharov, 1968), who used the Hamiltonian formalism for a description of wave propagation in deep water; see also Benney and Newell (1967). Hasimoto and Ono (1972) and Davey (1972) obtained the same result independently. Like Benney and
Newell (1967), they used the method of multiple-scale expansions in Euler coordinates. Yuen and Lake (1975), in turn, derived the NLS equation on the basis of the averaged Lagrangian method. Benney and Roskes (1969) extended those two-dimensional theories to the case of three-dimensional wave perturbations in a finite-depth fluid and obtained equations that are now known as the Davey-Stewartson equations. In this particular case, the equation proves the existence of the transverse instability of a plane wave, which is much stronger than a longitudinal one. This circumstance diminishes the role and meaning of the NLS equation for sea applications. Meanwhile, the one-dimensional NLS equation has been successfully tested many times in laboratory wave tanks, and natural observations have been compared with numerical calculations in the framework of this equation.

In all the cited papers, wave motion was considered to be potential. However, wave formation and propagation frequently occur against the background of a shear flow possessing vorticity. Wave train modulations upon arbitrary vertically sheared currents were studied by Benney and Maslowe (1975). Using the method of multiple scales, Johnson (1976) examined the slow modulation of a harmonic wave moving at the surface of an arbitrary shear flow with a velocity profile $U(y)$, where $y$ is the vertical coordinate. He derived the NLS equation with coefficients that depend in a complicated way on a shear flow (Johnson, 1976). Oikawa et al. (1987) considered the properties of instability in weakly nonlinear three-dimensional wave packets in the presence of a shear flow. Their simultaneous equations reduce to the known NLS equation for the case of purely two-dimensional 
wave evolution. Li et al. (1987) and Baumstein (1998) studied the modulation instability of the Stokes wave train and derived an NLS equation for a uniform shear flow in deep water when $U(y)=\Omega_{0} y$ and $\Omega_{z}=\Omega_{0}$ is constant vorticity ( $z$ is the horizontal coordinate normal to the $x, y$ plane of the flow; the wave propagates in the $x$ direction).

Thomas et al. (2012) generalized their results for a finitedepth fluid and confirmed that a linear shear flow may significantly modify the stability properties of weakly nonlinear Stokes waves. In particular, for the waves propagating in the direction of the flow, the Benjamin-Feir (modulational) instability can vanish in the presence of positive vorticity $\left(\Omega_{0}<0\right)$ for any depth.

In the traditional Eulerian approach to the propagation of weakly nonlinear waves against the background current, a shear flow determines vorticity in a zero approximation. Depending on the flow profile $U(y)$, it may be arbitrary and equal to $-U^{\prime}(y)$. At the same time, the vorticity of wave perturbations $\Omega_{n}, n \geq 1$, i.e., the vorticity in the first and subsequent approximations in the wave steepness parameter $\varepsilon=k A_{0}$ ( $k$ is wave number and $A_{0}$ is wave amplitude), depends on its form. In Eulerian coordinates, the vorticity of wave perturbations is a function not only of $y$, but of $x$ and $t$ variables as well. Plane waves on a shear flow with a linear vertical profile are regarded to be an exception ( $\mathrm{Li}$ et al., 1987; Baumstein, 1998; Thomas et al., 2012). For such waves the vorticity is constant in a zero approximation, and all the vorticities in wave perturbations are equal to zero. For an arbitrary vertical profile of the shear flow (Johnson, 1976), expressions for the functions $\Omega_{n}$ can hardly be predicted even qualitatively.

The Lagrangian method allows for the application of a different approach. In the plane flow, the vorticity of fluid particles is preserved and can be expressed via Lagrangian coordinates only. Thus, not only the vertical profile of the shear flow defining the vorticity in a zero approximation, but also the expressions for the vorticity of the following orders of smallness can be arbitrary. The expression for the vorticity is written in the form

$\Omega(a, b)=-U^{\prime}(b)+\sum_{n \geq 1} \varepsilon^{n} \Omega_{n}(a, b)$,

where $a, b$ are the horizontal and vertical Lagrangian coordinates, respectively, $U(b)$ is the vertical profile of the shear flow, and the particular conditions for defining the $\Omega_{n}$ functions can be found. For the given shear flow, this approach allows for the study of wave perturbations under the most general law of the distribution of vorticities $\Omega_{n}$. In this paper, we do not consider shear flow and vorticity in the linear approximation $\left(U=0 ; \Omega_{1}=0\right)$, whereas vorticity in the quadratic approximation is an arbitrary function. This corresponds to the rotational flow proportional to $\varepsilon^{2}$. We can define both the shear flow and the localized vortex.

The dynamics of plane wave trains on the background flows with arbitrary low vorticity have not been studied be- fore. The idea to study wave trains with quadratic (with respect to the wave steepness parameter) vorticity was realized earlier for the spatial problems in the Euler variables. Hjelmervik and Trulsen (2009) derived the NLS equation for vorticity distribution as

$\Omega_{y} / \omega=O\left(\varepsilon^{2}\right), \quad\left(\Omega_{x}, \Omega_{z}\right) / \omega=O\left(\varepsilon^{3}\right)$,

where $\omega$ is the wave frequency. The vertical vorticity of wave perturbations exceeds the other two vorticity components by a factor of 10 . This vorticity distribution corresponds to the low (of order $\varepsilon$ ) velocity of the horizontally inhomogeneous shear flow. Hjelmervik and Trulsen (2009) used the NLS equation to study the statistics of rogue waves on narrow current jets, and Onorato et al. (2011) used that equation to study the opposite flow rogue waves. The effect of low vorticity ( $\varepsilon^{2}$ order of magnitude) in the paper by Hjelmervik and Trulsen (2009) is reflected in the NLS equation. This fact, like the NLS nonlinear term for plane potential waves, may be attributed to the presence of an average current nonuniform over the fluid depth.

Colin et al. (1995) considered the evolution of threedimensional vortex disturbances in a finite-depth fluid for a different type of vorticity distribution:

$\Omega_{y}=0, \quad\left(\Omega_{x}, \Omega_{z}\right) / \omega=O\left(\varepsilon^{2}\right)$.

They reduced the problem to a solution for the DaveyStewartson equations by means of the multiple-scale expansion method in Eulerian variables. In this case, vorticity components are calculated after the solution to the problem. Similarly to the traditional Eulerian approach (Johnson, 1976), the form of the quadratic vorticity distribution is very special and does not cover all of its numerous possible distributions.

In this paper, we consider the plane problem of nonlinear wave packets propagating in an ideal incompressible fluid with the following form of vorticity distribution:

$\Omega_{z} / \omega=O\left(\varepsilon^{2}\right)$.

In contrast to Hjelmervik and Trulsen (2009), Onorato et al. (2011), and Colin et al. (1996), the flow is twodimensional $\left(\Omega_{x}=\Omega_{y}=0\right)$. The propagation of a packet of potential waves gives rise to a weak counterflow underneath the free water surface with velocity proportional to the square of the wave steepness (McIntyre, 1982). In the considered problem, this potential flow is superimposed with the rotational one of the same order of magnitude. This results in the appearance of an additional term in the NLS equation and in a change of the coefficient in the nonlinear term. So, the difference from the NLS solutions derived for a strictly potential fluid motion was revealed.

The examination is made in the Lagrangian variables. The Lagrangian variables are rarely used in fluid mechanics because of a more complex type of nonlinear equation 
in Lagrangian form. However, when considering the vortexinduced oscillations of a free fluid surface, the Lagrangian approach has two major advantages. First, unlike the Euler description method, the shape of the free surface is known and determined by the condition of the equality to zero $(b=$ 0 ) of the vertical Lagrangian coordinate. Second, the vortical motion of liquid particles is confined within the plane and is a function of Lagrangian variables $\Omega_{z}=\Omega_{z}(a, b)$, so the type of vorticity distribution in the fluid can be preset. The Eulerian approach does not allow this. In this case, the secondorder vorticity is defined as a known function of Lagrangian variables.

Here, hydrodynamic equations are solved in Lagrangian form through the multiple-scale expansion method. A nonlinear Schrödinger equation with variable coefficients is derived. Possible ways of reducing it to the NLS equation with constant coefficients are studied.

The paper is organized as follows. Section 2 describes the Lagrangian approach to studying wave oscillations at the free surface of a fluid. The zero of the Lagrangian vertical coordinate corresponds to the free surface, thus simplifying the formulation of the pressure boundary conditions. The specific feature of the proposed approach is the introduction of a complex coordinate of a fluid particle trajectory. In Sect. 3, a nonlinear evolution equation is derived on the basis of the method of multiple-scale expansion. Different solutions to the NLS equation adequately describing various examples of vortex waves are considered in Sect. 4 . The transform from of the Lagrangian coordinates to the Euler description of the solutions to the NLS equation is shown in Sect. 5. Section 6 summarizes the obtained results.

\section{Basic equations in Lagrangian coordinates}

Consider the propagation of a packet of gravity surface waves in a rotational infinitely deep fluid. Two-dimensional hydrodynamic equations of an incompressible inviscid fluid in Lagrangian coordinates have the following form (Lamb, 1932; Abrashkin and Yakubovich, 2006; Bennett, 2006):

$\frac{D(X, Y)}{D(a, b)}=[X, Y]=1$,

$X_{t t} X_{a}+\left(Y_{t t}+g\right) Y_{a}=-\frac{1}{\rho} p_{a}$,

$X_{t t} X_{b}+\left(Y_{t t}+g\right) Y_{b}=-\frac{1}{\rho} p_{b}$

where $X, Y$ are the horizontal and vertical Cartesian coordinates, $a, b$ are the horizontal and vertical Lagrangian coordinates of fluid particles, $t$ is time, $\rho$ is fluid density, $p$ is pressure, $g$ is acceleration due to gravity, and the subscripts mean differentiation with respect to the corresponding variable. The square brackets denote the Jacobian. The $b$ axis is directed upwards, and $b=0$ corresponds to the free surface. Equation (1) is a volume conservation equation. Equa-

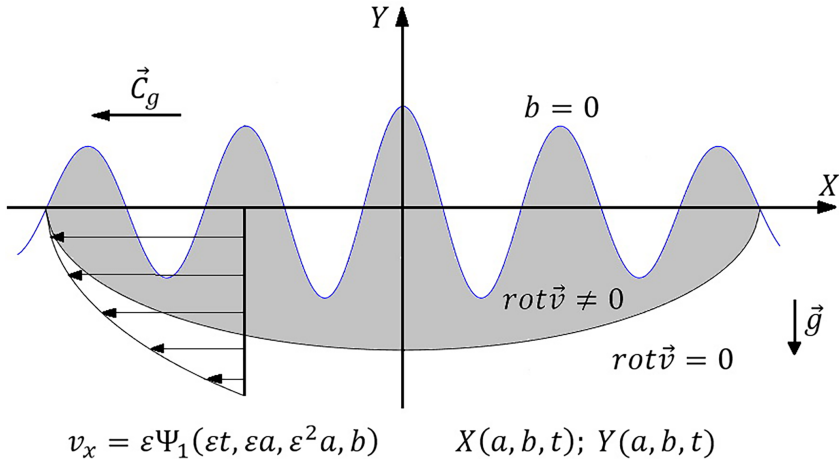

Figure 1. Problem geometry: $v_{x}$ is the average current.

tions (2) and (3) are momentum equations. The geometry of the problem is presented in Fig. 1.

By making use of cross differentiation, it is possible to exclude pressure and obtain the condition of the conservation of vorticity along the trajectory (Lamb, 1932; Abrashkin and Yakubovich, 2006; Bennett, 2006):

$X_{t a} X_{b}+Y_{t a} Y_{b}-X_{t b} X_{a}-Y_{t b} Y_{a}=\Omega(a, b)$.

This equation is equivalent to the momentum Eqs. (2) and (3) but involves the explicit vorticity of liquid particles, $\Omega$, which in the case of two-dimensional flows is the function of Lagrangian coordinates only.

We introduce a complex coordinate of a fluid particle trajectory $W=X+i Y \quad(\bar{W}=X-i Y)$, where the overline means complex conjugation. In the new variables, Eqs. (1) and (4) take the form

$$
\begin{aligned}
& {[W, \bar{W}]=-2 i,} \\
& \operatorname{Re}\left[W_{t}, \bar{W}\right]=\Omega(a, b) .
\end{aligned}
$$

After simple algebraic manipulations, Eqs. (2) and (3) reduce to the following single equation:

$W_{t t}=-i g+i \rho^{-1}[p, W]$.

Equations (5) and (6) will be further used to find the coordinates of the complex trajectories of fluid particles, and Eq. (7) determines the pressure of the fluid. The boundary conditions are the non-flowing condition at the bottom $\left(Y_{t} \rightarrow 0\right.$ at $b \rightarrow-\infty)$ and constant pressure at the free surface (at $b=0)$.

The Lagrangian coordinates mark the position of fluid particles. In the Eulerian description, the displacement of the free surface $Y_{\mathrm{S}}(X, t)$ is calculated in an explicit form, but in the Lagrangian description it is defined parametrically by the following equalities: $Y_{\mathrm{S}}(a, t)=Y(a, b=0, t)$ and $X_{\mathrm{S}}(a, t)=X(a, b=0, t)$, where the Lagrangian horizontal coordinate $a$ plays the role of a parameter. Its value along the free surface $b=0$ varies in the $(-\infty ; \infty)$ range. In Lagrangian coordinates, the function $Y_{\mathrm{s}}(a, t)$ defines the displacement of the free surface. 


\section{Derivation of evolution equation}

Let us represent the function $W$ using the multiple-scale method in the following form:

$$
\begin{aligned}
& W=a_{0}+i b+w\left(a_{l}, b, t_{l}\right), \\
& \quad a_{l}=\varepsilon^{l} a, \quad t_{l}=\varepsilon^{l} t ; \quad l=0,1,2,
\end{aligned}
$$

where $\varepsilon$ is the small parameter of wave steepness. All unknown functions and the given vorticity can be represented as a series in this parameter:

$$
\begin{aligned}
& w=\sum_{n=1} \varepsilon^{n} w_{n} ; p=p_{0}-\rho g b+\sum_{n=1} \varepsilon^{n} p_{n} ; \\
& \Omega=\sum_{n=1} \varepsilon^{n} \Omega_{n}(a, b) .
\end{aligned}
$$

In the formula for the pressure, the term with hydrostatic pressure is selected, and $p_{0}$ is the constant atmospheric pressure at the fluid surface. The representations (8) and (9) are substituted into Eqs. (5)-(7).

\subsection{Linear approximation}

In a first approximation in the small parameter, we have the following system of equations:

$$
\begin{aligned}
& \operatorname{Im}\left(i w_{1 a_{0}}+w_{1 b}\right)=0, \\
& \operatorname{Re}\left(i w_{1 a_{0}}+w_{1 b}\right)_{t_{0}}=-\Omega_{1}, \\
& w_{1 t_{0} t_{0}}+\rho^{-1}\left(p_{1 a_{0}}+i p_{1 b}\right)=i g w_{1 a_{0}} .
\end{aligned}
$$

The solution satisfying the continuity Eq. (10) and the equation of the conservation of vorticity (11) describes a monochromatic wave (for definiteness, we consider the wave propagating to the left) and the average horizontal current:

$$
\begin{aligned}
w_{1}= & A\left(a_{1}, a_{2}, t_{1}, t_{2}\right) \exp \left[i\left(k a_{0}+\omega t_{0}\right)+k b\right] \\
& +\psi_{1}\left(a_{1}, a_{2}, b, t_{1}, t_{2}\right), \quad \Omega_{1}=0 .
\end{aligned}
$$

Here, $A$ is the complex amplitude of the wave, $\omega$ is its frequency, and $k$ is the wave number. The function $\psi_{1}$ is real and will be found in the next approximation.

The substitution of solution (13) into Eq. (12) yields the equation for the pressure,

$\rho^{-1}\left(p_{1 a_{0}}+i p_{1 b}\right)=\left(\omega^{2}-g k\right) A \exp \left[i\left(k a_{0}+\omega t_{0}\right)+k b\right]$,

which is solved analytically as

$$
\begin{aligned}
p_{1}= & -\operatorname{Re} \frac{i\left(\omega^{2}-g k\right)}{k} \rho A \exp \left[i\left(k a_{0}+\omega t_{0}\right)+k b\right] \\
& +C_{1}\left(a_{1}, a_{2}, t_{1}, t_{2}\right),
\end{aligned}
$$

where $C_{1}$ is an arbitrary function. The boundary condition at the free surface is $\left.p_{1}\right|_{b=0}=0$, which leads to $\omega^{2}=g k$ and $C_{1}=0$. Thus, in the first approximation the pressure correction $p_{1}$ is equal to zero.

\subsection{Quadratic approximation}

The equations of the second order of the perturbation theory can be written as follows:

$$
\begin{aligned}
& \operatorname{Im}\left(i w_{2 a_{0}}+w_{2 b}+i w_{1 a_{1}}-w_{1 a_{1}} \overline{w_{1 b}}\right)=0, \\
& \operatorname{Re}\left[i w_{2 t_{0} a_{0}}+w_{2 t_{0} b}+i\left(w_{1 t_{0} a_{1}}+w_{1 t_{1} a_{0}}\right)\right. \\
& \left.\quad-w_{1 t_{0} a_{0}} \overline{w_{1 b}}+w_{1 t_{1} b}+w_{1 t_{0} b} \overline{w_{1 a_{0}}}\right]=-\Omega_{2}, \\
& w_{2 t_{0} t_{0}}+\rho^{-1}\left(p_{2 a_{0}}+i p_{2 b}\right)=i g\left(w_{2 a_{0}}+w_{a_{1}}\right) \\
& \quad-2 w_{1 t_{1} t_{0}} .
\end{aligned}
$$

By substituting expression (13) for $w_{1}$ into Eq. (16), we obtain

$$
\begin{gathered}
\operatorname{Im}\left[i w_{2 a_{0}}+w_{2 b}-i\left(k \psi_{1 b} A-A_{a_{1}}\right) \exp \left[i\left(k a_{0}+\omega t_{0}\right)+k b\right]\right. \\
\left.\quad-i k^{2}|A|^{2} e^{2 k b}+i \psi_{1 a_{1}}\right]=0
\end{gathered}
$$

which is integrated as follows:

$$
\begin{aligned}
w_{2}= & i\left(k A \psi_{1}-b A_{a_{1}}\right) \exp \left[i\left(k a_{0}+\omega t_{0}\right)+k b\right] \\
& +\psi_{2}+i f_{2},
\end{aligned}
$$

where $\psi_{2}, f_{2}$ are the functions of slow coordinates and the Lagrangian vertical coordinate $b$, and

$$
f_{2 b}=k^{2}|A|^{2} \exp 2 k b-\psi_{1 a_{1}},
$$

where $\psi_{2}$ is an arbitrary real function. It will be determined in a solution in the cubic approximation.

When Eqs. (13) and (20) are substituted into Eq. (17), the sum of the terms containing the exponential factor becomes equal to zero, and the remaining terms satisfy the equation

$\psi_{1 t_{1} b}=-2 k^{2} \omega|A|^{2} \exp (2 k b)-\Omega_{2}$.

The expression for the function $\psi_{1}$ can be found by simple integration. It should be emphasized that the vorticity in the second approximation, which is part of Eq. (22), is an arbitrary function of slow horizontal and vertical Lagrangian coordinates so that $\Omega_{2}=\Omega_{2}\left(a_{1}, a_{2}, b\right)$.

Taking into account the solutions in the first two approximations, we can write Eq. (18) as

$$
\begin{aligned}
& \rho^{-1}\left(p_{2 a_{0}}+i p_{2 b}\right)=i\left(g A_{a_{1}}-2 \omega A_{t_{1}}\right) \exp \left[i\left(k a_{0}+\omega t_{0}\right)+k b\right] \\
& \quad+i g \psi_{1 a_{1}} .
\end{aligned}
$$

Its solution determines the pressure correction:

$$
\begin{aligned}
p_{2}= & R e\left[\frac{1}{k}\left(g A_{a_{1}}-2 \omega A_{t_{1}}\right) \exp \left[i\left(k a_{0}+\omega t_{0}\right)+k b\right]\right] \\
& +\rho g \int_{0}^{b} \psi_{1 a_{1}} \mathrm{~d} b+C_{2}\left(a_{1}, a_{2}, t_{1}, t_{2}\right) .
\end{aligned}
$$

The integration limits in the penultimate term are chosen so that this integral term equals zero at the free surface. Due to 
the boundary condition for pressure, $\left(p_{2}(b=0)=0\right), C_{2}=$ 0 , and

$A_{t_{1}}-c_{\mathrm{g}} A_{a_{1}}=0 ; \quad c_{\mathrm{g}}=\frac{g}{2 \omega}=\frac{1}{2} \sqrt{\frac{g}{k}}$,

where $c_{\mathrm{g}}$ is the group velocity of wave propagation in deep water, which in this approximation is independent of fluid vorticity. As expected, in this approximation the wave moves with group velocity $c_{\mathrm{g}}$ to the left (the "minus" sign in Eq. 24).

\subsection{Cubic approximation}

The equation of continuity and the condition of the conservation of vorticity in the third approximation are written in the form

$$
\begin{aligned}
& \operatorname{Im}\left[i w_{2 a_{0}}+w_{3 b}+i\left(w_{1 a_{2}}+w_{2 a_{1}}+w_{2 a_{0}}\right)\right. \\
& \left.\quad-\left(w_{1 a_{1}}+w_{2 a_{2}}\right) \overline{w_{1 b}}-w_{1 a_{0}} \overline{w_{2 b}}\right]=0, \\
& \operatorname{Re}\left[i w_{3 t_{0} a_{0}}+w_{3 t_{0} b}+i\left(w_{1 t_{2} a_{0}}+w_{1 t_{1} a_{1}}+w_{1 t_{0} a_{2}}+w_{2 t_{1} a_{0}}\right.\right. \\
& \left.\quad+w_{2 t_{0} a_{1}}\right)+w_{1 t_{2} b}-\overline{w_{2 b}} w_{1 t_{0} a_{0}}- \\
& +w_{2 t_{1} b}-w_{1 b}\left(w_{1 t_{0} a_{1}}+w_{1 t_{1} a_{0}}+w_{2 t_{0} a_{0}}\right)+ \\
& \left.\quad+\overline{w_{1 a_{0}}}\left(w_{1 t_{1} b}+w_{2 t_{0} b}\right)+w_{1 t_{0} b}\left(\overline{w_{1 a_{1}}}+\overline{w_{2 a_{0}}}\right)\right]=-\Omega_{3} .
\end{aligned}
$$

We substitute the solutions in the first and second approximations into the simultaneous equations:

$$
\begin{aligned}
& \operatorname{Im}\left[i w_{3 a_{0}}+w_{3 b}+i\left(\psi_{1 a_{2}}+\psi_{2 a_{1}}\right)+2 k(k b+1) A \overline{A_{a_{1}}} e^{2 b}\right. \\
& \left.\quad+G_{b} e^{i\left(k a_{0}+\omega t_{0}\right)+k b}\right]=0 \\
& \operatorname{Re}\left\{\left[i w_{3 a_{0}}+w_{3 b}+\left(G_{b}+2 k \psi_{1 t_{1} b} \omega^{-1} A\right) e^{i\left(k a_{0}+\omega t_{0}\right)+k b}\right]_{t_{0}}\right. \\
& \quad+\psi_{2 t_{1} b}+\psi_{1 t_{2} b}+ \\
& \left.\quad+i \omega k(4 k b+5) A \overline{A_{a_{1}}} e^{2 k b}\right\}=-\Omega_{3} \\
& G=i b A_{a_{2}}+\frac{b^{2}}{2} A_{a_{1} a_{1}}-(k b+1) \psi_{1} A_{a_{1}} \\
& \quad-\left(i k \psi_{2}+k f_{2}-\frac{k^{2}}{2} \psi_{1}^{2}\right) A
\end{aligned}
$$

We seek a solution for the third approximation in the following form:

$$
\begin{aligned}
w_{3}= & \left(G_{1}-G\right) e^{i\left(k a_{0}+\omega t_{0}\right)+k b}+G_{2} e^{-i\left(k a_{0}+\omega t_{0}\right)+k b} \\
& +\psi_{3}+i f_{3},
\end{aligned}
$$

where $G_{1}, G_{2}, \psi_{3}$, and $f_{3}$ are functions of slow coordinates and $b$. By substituting this expression into Eqs. (28) and (29), we immediately find

$$
\begin{aligned}
& f_{3 b}+\psi_{2 a_{1}}+\psi_{1 a_{2}}+k(k b+1)\left(A \overline{A_{a_{1}}}-\bar{A} A_{a_{1}}\right) e^{2 k b}=0, \\
& \psi_{2 t_{1} b}+\psi_{1 t_{2} b}+\frac{1}{2}(4 k b+5) \omega k\left(A \overline{A_{a_{1}}}-\bar{A} A_{a_{1}}\right) e^{2 k b}=-\Omega_{3} .
\end{aligned}
$$

The function $\psi_{2}$ according to Eq. (33) is determined by known solutions for $A$ and $\psi_{1}$ and by the given distribution of
$\Omega_{3}$. The expression for the function $f_{3}$ is then derived from Eq. (32). These functions determine the horizontal and vertical average motion, respectively. But in this approximation they are not included in the evolution equation for the wave envelope. The function $\psi_{3}$ will be found in the next approximation.

When solving Eqs. (28) and (29), we found

$$
\begin{aligned}
& G_{1}=-k \omega^{-1} \psi_{1 t_{1}} A, \\
& G_{2}=k \omega^{-1}\left(2 k e^{-2 k b} \int_{-\infty}^{b} \psi_{1 t_{1}} e^{2 k b^{\prime}} \mathrm{d} b^{\prime}-\psi_{1 t_{1}}\right) \bar{A} .
\end{aligned}
$$

These relationships should be substituted into Eq. (7), which in this approximation has the form

$$
\begin{aligned}
& w_{3 t_{0} t_{0}}-i g w_{3 a_{0}}=i \rho^{-1}\left[i\left(p_{2 a_{1}}+p_{3 a_{0}}\right)-p_{3 b}-p_{2 b} w_{1 a_{0}}\right. \\
& \left.\quad+\rho g\left(w_{1 a_{2}}+w_{2 a_{1}}\right)\right]--2 w_{1 t_{2} t_{0}}-w_{1 t_{1} t_{1}}-2 w_{2 t_{0} t_{1}} .
\end{aligned}
$$

Taking into account Eqs. (13), (20), (24), (31), and (34), we rewrite it as follows:

$$
\begin{aligned}
& \rho^{-1}\left(p_{3 a_{0}}+i p_{3 b}\right)=\left(-2 i \omega \frac{\partial A}{\partial t_{2}}+i g \frac{\partial A}{\partial a_{2}}-\frac{\partial^{2} A}{\partial t_{1}^{2}}+2 \omega k \psi_{1 t_{1} A} A\right) \\
& e^{i\left(k a_{0}+\omega t_{0}\right)+k b}+ \\
& +2 \omega^{2} G_{2} \bar{A} e^{-i\left(k a_{0}+\omega t_{0}\right)+k b}+i g\left(\psi_{2 a_{1}}+\psi_{1 a_{2}}\right)+I \\
& I=-g\left(f_{2 a_{1}}-\int_{b}^{0} \psi_{1 a_{1} a_{1} \mathrm{~d} b}\right)-\psi_{t_{1} t_{1}} .
\end{aligned}
$$

By virtue of the relationships (21), (22), and (25), the derivative of $I$ along the vertical Lagrangian coordinate is zero $\left(I_{b}=0\right)$, so $I$ is the only function of the slow coordinates and time $-a_{l}, t_{l}, l \geq 1$. The contribution of the term $I\left(a_{l}, t_{l}\right) \neq 0$ to the pressure is complex, so it demands $I=0$.

The solution to Eq. (36) yields the expression for the pressure perturbation in the third approximation:

$$
\begin{aligned}
\frac{p_{3}}{\rho} & =\operatorname{Reik}^{-1}\left(2 i \omega \frac{\partial A}{\partial t_{2}}-i g \frac{\partial A}{\partial a_{2}}+\frac{\partial^{2} A}{\partial t_{1}^{2}}\right. \\
& \left.-4 \omega k^{2} A e^{-2 k b} \int_{-\infty}^{b} \psi_{1 t_{1}} e^{2 k b^{\prime}} \mathrm{d} b^{\prime}\right) e^{i\left(k a_{0}+\omega t_{0}\right)+k b}+ \\
& +\rho g \int_{0}^{b}\left(\psi_{2 a_{1}}+\psi_{1 a_{2}}\right) \mathrm{d} b^{\prime} .
\end{aligned}
$$

In Eq. (37), the integration limits for the second integral term have been preset to satisfy the boundary condition at the free surface (the pressure $p_{3}$ should turn to zero). Then the factor before the exponent should be equal to zero:

$$
2 i \omega \frac{\partial A}{\partial t_{2}}-i g \frac{\partial A}{\partial a_{2}}+\frac{\partial^{2} A}{\partial t_{1}^{2}}-4 \omega k^{2} A \int_{-\infty}^{0} \psi_{1 t_{1}} e^{2 k b} \mathrm{~d} b=0 .
$$


By introducing the "running" coordinate $\zeta_{2}=a_{2}+c_{\mathrm{g}} t_{2}$, we can reduce Eq. (38) to a compact form:

$i \frac{\partial A}{\partial a_{2}}-\frac{k}{\omega^{2}} \frac{\partial^{2} A}{\partial t_{1}^{2}}+\frac{4 k^{3} A}{\omega} \int_{-\infty}^{0} \psi_{1 t_{1}} e^{2 k b} \mathrm{~d} b=0$.

Further, it will be shown that the variables in Eqs. (38) and (39) have been chosen so that they could be easily reduced (under particular assumptions) to the classical NLS equation.

The explicit form of the function $\psi_{1 t_{1}}$ is found by integrating Eq. (22):

$\psi_{1 t_{1}}=-k \omega|A|^{2} e^{2 k b}-\int_{-\infty}^{b} \Omega_{2}\left(a_{2}, b^{\prime}\right) \mathrm{d} b^{\prime}-U\left(a_{2}, t_{1}\right)$.

This expression includes three terms. All of them describe a certain component of the average current. The first one is proportional to the square of the amplitude modulus and describes the classical potential drift of fluid particles (see Henderson et al., 1999, for example). The second one is caused by the presence of low vorticity in the fluid. Finally, the third item, including $U\left(a_{2}, t_{1}\right)$, describes an additional potential flow. It appears in the integration of Eq. (22) over the vertical coordinate $b$ and will evidently not disappear in the case of $A=0$. This is a certain external flow that is chosen depending on a specific problem. Note that a term of that kind arises in the Eulerian description of potential wave oscillations of the free surface as well. In the paper by Stocker and Peregrine (1999), $U=U_{*} \sin (k x-\omega t)$ was chosen and interpreted as a harmonically changing surface current induced by an internal wave. We shall further take $U=0$.

After the substitution of Eq. (40), Eq. (39) may be written in the final form

$$
\begin{gathered}
i \frac{\partial A}{\partial a_{2}}-\frac{k}{\omega^{2}} \frac{\partial^{2} A}{\partial t_{1}^{2}}-k\left(k^{2}|A|^{2}+\beta\left(a_{2}\right)\right) A=0, \\
\beta\left(a_{2}\right)=\frac{4 k^{2}}{\omega} \int_{-\infty}^{0} e^{2 k b}\left(\int_{-\infty}^{b} \Omega_{2}\left(a_{2}, b^{\prime}\right) \mathrm{d} b^{\prime}\right) \mathrm{d} b .
\end{gathered}
$$

It is the nonlinear Schrödinger equation for the packet of surface gravity waves propagating in the fluid with vorticity distribution $\Omega=\varepsilon^{2} \Omega_{2}\left(a_{2}, b\right)$. The function $\Omega_{2}\left(a_{2}, b\right)$ determining flow vorticity may be an arbitrary function setting the initial distribution of vorticity. On integrating it twice, we find the vortex component of the average current, which is in no way related to the average current induced by the potential wave.

\section{Examples of the waves}

Let us consider some special cases following from Eq. (41).

\subsection{Potential waves}

In this case, $\Omega_{2}=0$ and Eq. (41) becomes the classical nonlinear Schrödinger equation for waves in deep water. Three kinds of analytical solutions to the NLS equation are usually discussed regarding water waves. The first one is the Peregrine breather propagated in space and time (Peregrine, 1983). This wave may be considered as a long wave limit of a breather, which is a pulsating mode of infinite wavelength (Grimshaw et al., 2010). The two others are the Akhmediev breather, which is the solution periodic in space and localized in time (Akhmediev et al., 1985), and the Kuznetsov-Ma breather, which is the solution periodic in time and localized in space (Kuznetsov, 1977; Ma, 1979). Both of the latter solutions evolve against the background of an unperturbed sine wave.

\subsection{Gerstner waves}

The exact Gerstner solution in complex form is written as (Lamb, 1932; Abrashkin and Yakubovich, 2006; Bennett, 2006)

$W=a+i b+i A \exp [i(k a+\omega t)+k b]$.

It describes a stationary traveling rotational wave with a trochoidal profile. Its dispersion characteristic coincides with the dispersion of linear waves in deep water $\omega^{2}=g k$. The fluid particles move in circles and there is no drift current.

Equation (42) is the exact solution to the problem. Following Eqs. (8) and (9), the Gerstner wave should be written as

$W=a_{0}+i b+\sum_{n \geq 1} \varepsilon^{n} \cdot i A \exp \left[i\left(k a_{0}+\omega t_{0}\right)+k b\right]$.

All of the functions $w_{n}$ in Eqs. (8) and (9) have the same form. To derive the vorticity of the Gerstner wave, Eq. (43) should be substituted into Eq. (6). Then one can find that in the linear approximation, the Gerstner wave is potential $\left(\Omega_{1}=0\right)$, but in the quadratic approximation it possesses vorticity:

$$
\Omega_{2 \mathrm{Gerstner}}=-2 \omega k^{2}|A|^{2} e^{2 k b} .
$$

For this type of vorticity distribution, the sum of the first two terms in the parentheses in Eq. (41) is equal to zero. From the physical point of view, this is due to the fact that the average current induced by the vorticity compensates exactly for the potential drift. The packet of weakly nonlinear Gerstner waves in this approximation is not affected by their nonlinearity, and the effect of the modulation instability for the Gerstner wave does not occur.

Generally speaking, this result is quite obvious. As there is no particle drift in the Gerstner wave, the function $\psi_{1}$ equals zero. So, the multiplier of the wave amplitude in Eqs. (38) and (39) may be neglected without finding the vorticity of the Gerster wave. 
Let us consider some particular consequences of the obtained result. For the irrotational $\left(\Omega_{2}=0\right)$ stationary $(A=$ $|A|=$ const) wave, Eq. (40) for the velocity of the drifting flow takes the form

$\psi_{1 t_{1}}=-\omega k A^{2} e^{2 k b}$.

It coincides with the expression for the Stokes drift in Lagrangian coordinates (in the Eulerian variables the profile of the Stokes current may be obtained by the substitution of $b$ for $y$ ). Thus, our result may be interpreted as a compensation of the Stokes drift by the shear flow induced by the Gerstner wave in a quadratic approximation. This conclusion is also fair in the "differential" formulation for vorticities. From Eq. (22), it follows that the vorticity of the Stokes drift equals the vorticity of the Gerstner wave with the inverse sign.

The absence of a nonlinear term in the NLS equation for the Gerstner waves obtained here in the Lagrangian formulation is a robust result and should appear in the Euler description as well. This follows from the famous Lighthill criterion for the modulation instability because the dispersion relation for the Gerstner wave is linear and does not include terms proportional to the wave amplitude.

\subsection{Gouyon waves}

As shown by Dubreil-Jacotin (1934), the Gerstner wave is a special case of a wide class of stationary waves with vorticity $\Omega=\varepsilon \Omega_{*}(\psi)$, where $\Omega_{*}$ is an arbitrary function and $\psi$ is a stream function. Those results were later developed by Gouyon (1958), who explicitly represented the vorticity in the form of a power series $\Omega=\sum_{n=1}^{\infty} \varepsilon^{n} \Omega_{n}(\psi)$ (see also the monograph by Sretensky, 1977).

When a plane steady flow is considered in the Lagrangian variables, the stream lines $\psi$ coincide with the isolines of the Lagrangian vertical coordinate $b$ (Abrashkin and Yakubovich, 2006; Bennett, 2006). We consider a steadystate wave at the surface of indefinitely deep water. Assume that there is no undisturbed shear current, but the wave disturbances have vorticity. Then, the formula for the vorticity is written as $\Omega=\sum_{n=1}^{\infty} \varepsilon^{n} \Omega_{n}(b)$. Here we will refer to the steady-state waves propagating in such a low-vorticity fluid as Gouyon waves. The properties of the Gouyon wave for the first two approximations were studied by Abrashkin and Zen'kovich (1990) in the Lagrangian description.

In our case, $\Omega_{1}=0$ and $\Omega_{2} \neq 0$; assuming the function $\Omega_{2}$ to be independent of the coordinate $a$, we can describe the Gouyon waves. The vorticity $\Omega_{2}$ depends on the coordinate $b$ only and has the following form:

$\Omega_{2 \text { Goyuon }}=\omega k^{2}|A|^{2} H(k b)$,

where $H(k b)$ is an arbitrary function. In the case of $H(k b)=$ $-2 \exp (2 k b)$, the vorticities of the Gerstner and Gouyon waves in the quadratic approximation coincide (compare Eqs. 44 and 46). In the considered approximation, the Gouyon wave generalizes the Gerstner wave. From Eq. (22), it follows that the function $\psi_{t_{1}}$ is equal to zero only when the vorticity of the Gouyon wave is equal to the vorticity of the Gerstner wave. Except for this case, the average current $\psi_{t_{1}}$ will always be present in the modulated Gouyon waves.

The substitution of the ratio (46) into Eq. (41) yields the NLS equation for the modulated Gouyon wave:

$$
\begin{gathered}
i \frac{\partial A}{\partial a_{2}}-\frac{k}{\omega^{2}} \frac{\partial^{2} A}{\partial t_{1}^{2}}-\beta_{\mathrm{G}} k^{3}|A|^{2} A=0, \\
\beta_{\mathrm{G}}=1+4 \int_{-\infty}^{0} e^{2 \widetilde{b}}\left(\int_{-\infty}^{\widetilde{b}} H\left(\widetilde{b^{\prime}}\right) \mathrm{d} \widetilde{b^{\prime}}\right) \mathrm{d} \widetilde{b}, \quad \widetilde{b}=k b,
\end{gathered}
$$

where $\widetilde{b}$ is a dimensionless vertical coordinate. The coefficient of the nonlinear term in the NLS equation varies when the wave vorticity is taken into account. For the Gerstner wave it may be equal to zero like for the Gouyon wave when the following condition is satisfied:

$\int_{-\infty}^{0} e^{2 \widetilde{b}}\left(\int_{-\infty}^{\widetilde{b}} H\left(\widetilde{b}^{\prime}\right) \mathrm{d} \widetilde{b}^{\prime}\right) \mathrm{d} \widetilde{b}=-\frac{1}{4}$.

Clearly, an infinite number of distributions of the vorticity $H(\widetilde{b})$ meeting this condition are possible. However, the realization of one of them seems hardly probable. In the real ocean, distributions of the vorticity with a certain sign of $\beta_{\mathrm{G}}$ are more likely to be implemented. Its negative values correspond to the defocusing NLS equation and the positive ones are related to the focusing NLS equation. In the latter case, the maximum value of the increment and the width of the modulation instability zone of a uniform train of vortex waves vary depending on the value of $\beta_{\mathrm{G}}$.

Equations (39) and (47) will be focusing for $\psi_{1 t_{1}}<0, b \leq$ 0 and defocusing if $\psi_{1 t_{1}}>0, b \leq 0$. The case of the signvariable function $\psi_{1 t_{1}}$ requires additional research. From the physical viewpoint, the sign of this function is defined by the ratio of the velocity of the Stokes drift (45) to the velocity of the current induced by the vorticity (the integral term in Eq. 40). For $\psi_{1 t_{1}}<0$, the Stokes drift either dominates over a vortex current or both of them have the same direction. When $\psi_{1 t_{1}}>0$, the vortex current dominates over the counter Stokes drift. In the case of the sign variable $\psi_{1 t_{1}}$, the ratio of these currents varies at different vertical levels, thereby requiring a direct calculation of $\beta_{\mathrm{G}}$.

\subsection{Waves with inhomogeneous vorticity distribution along both coordinates}

Neither a vorticity expression nor methods of its definition were discussed when deriving the NLS equation. Sections 4.2 and 4.3 are devoted to the problems of the Gerstner and Gouyon waves; the vorticity was set to be proportional to 
a square modulus of the wave amplitude. Note that waves can propagate against the background of some vortex current, for example, the localized vortex. In this case, the vorticity may be presented in the form

$\Omega_{2}\left(a_{2}, b\right)=\omega\left[\phi_{v}\left(a_{2}, b\right)+k^{2}|A|^{2} \phi_{\mathrm{w}}\left(a_{2}, b\right)\right]$,

where the function $\omega \phi_{v}$ defines the vorticity of the background vortex current and the function $\omega k^{2}|A|^{2} \phi_{\mathrm{w}}$ defines the vorticity of waves. In the most general case, both functions depend on the horizontal Lagrangian coordinate as well. Then, Eq. (41) takes the form

$$
\begin{gathered}
i \frac{\partial A}{\partial a_{2}}-\frac{k}{\omega^{2}} \frac{\partial^{2} A}{\partial t_{1}^{2}}-k \beta_{v}\left(a_{2}\right) A-k^{3}\left(1+\beta_{\mathrm{w}}\left(a_{2}\right)\right)|A|^{2} A=0 \\
\beta_{v, w}\left(a_{2}\right)=4 \int_{-\infty}^{0} e^{2 \widetilde{b}}\left(\int_{-\infty}^{\widetilde{b}} \phi_{v, w}\left(a_{2}, \widetilde{b}^{\prime}\right) \mathrm{d} \widetilde{b}^{\prime}\right) \mathrm{d} \widetilde{b}
\end{gathered}
$$

The substitution

$A *=A \exp \left(-i k \int_{-\infty}^{a_{2}} \beta_{v}\left(a_{2}\right) d a_{2}\right)$

reduces Eq. (49) to the NLS equation with a nonuniform multiplier for the nonlinear term:

$i \frac{\partial A^{*}}{\partial a_{2}}-\frac{k}{\omega^{2}} \frac{\partial^{2} A^{*}}{\partial t_{1}^{2}}-k^{3}\left(1+\beta_{\mathrm{w}}\left(a_{2}\right)\right)\left|A^{*}\right|^{2} A^{*}=0$.

Let us consider the propagation of the Gouyon wave when $\beta_{\mathrm{W}}=$ const $=\beta_{\mathrm{G}}-1$, and Eq. (51) turns into the classical NLS equation (47). As shown in Sect. 4.3, it describes the modulated Gouyon waves. Therefore, based on the substitution of Eq. (50) one can conclude that the propagation of the Gouyon waves against the background of the nonuniform vortex current results in the variation in the wave number of the carrier wave. For $\beta_{\mathrm{w}}=0$, Eq. (51) describes the propagation of a packet of potential waves against the background of the nonuniform weakly vortical current. The specific features of the wave propagation related to the variable $\beta_{\mathrm{w}}$ require special investigation.

\section{On the equivalence of Lagrangian and Eulerian approaches}

Consider the correlation between the Eulerian and the Lagrangian description of wave packets. To obtain the value for the elevation of the free surface we substitute the expressions (8), (9), and (13) and $b=0$ into the equation for $Y=\operatorname{Im} W$ written in the following form:

$Y_{L}=\varepsilon \operatorname{Im} A\left(a_{2}, t_{1}\right) \exp i\left(k a_{0}+\omega t_{0}\right)$, where $A\left(a_{2}, t_{1}\right)$ is the solution to Eq. (41). This expression defines the wave profile in Lagrangian coordinates. To rewrite this equation in the Eulerian variables, it is necessary to define $a$ via $X$. From the relation (8), it follows that

$X=a+\varepsilon \operatorname{Re}\left(w_{1}+\sum_{n=2} \varepsilon^{n-1} w_{n}\right)=a+O(\varepsilon)$,

and the elevation of the free surface in the Eulerian variables $Y_{E}$ will be written as

$$
\begin{aligned}
Y_{E}= & \varepsilon \operatorname{Im} A\left(X_{2}, t_{1}\right) \exp i\left(k X_{0}+\omega t_{0}\right)+O\left(\varepsilon^{2}\right), \\
& X_{l}=\varepsilon^{l} X .
\end{aligned}
$$

The coordinate $a$ plays the role of $X$, so the following substitutions are valid for the Lagrangian approach:

$a_{0} \rightarrow X_{0} ; \quad a_{1} \rightarrow X_{1} ; \quad a_{2} \rightarrow X_{2}$

This result may be called an "equivalence principle" between the Lagrange and the Euler descriptions for solutions in the linear approximation. This principle is valid for both the potential and rotational waves.

To express the solution to Eq. (41) in the Eulerian variables, it is necessary to use the equivalence principle and to replace the horizontal Lagrangian coordinate $a_{2}$ with the $X_{2}$ coordinate. So, there are no discrepancies between the Eulerian and the Lagrangian estimations of the NLS equation for the free surface elevation.

Taking this into account, we can conclude that the result will be the same in the Eulerian description if the vorticity $\Omega_{2}$ is a function of the $x, y$ coordinates. So, when studying the wave packet dynamics in the vortical liquid in the Eulerian variables, it is necessary to replace (for example, in Eqs. 41 and 51) the horizontal Lagrangian coordinate with the Eulerian one.

Equation (47) can also be derived in Eulerian variables. The key idea is to take into consideration a weak shear flow. This approach is similar to the method used in the paper by Hjelmervik and Trulsen (2009), where the wave propagates along a weak horizontal shear current. Shrira and Slunyaev (2014) used this technique to study trapped waves in a uniform jet stream. They derived the NLS equation for a single mode. Later, Slunyaev (2016) generalized the result to the case of a vortex jet flow. Our result was obtained with a weak vertical shear flow taken into account. In particular, to describe modulated Guyon waves, the Johnson approach (1976) should be modified, assuming a shear flow of the order of epsilon.

The solutions to the considered problem in the Lagrange and the Euler forms in the quadratic and cubic approximations differ from each other. To obtain a full solution in the Lagrange form, one should find the functions $\psi_{1}, \psi_{2}, \psi_{3}, f_{2}$, and $f_{3}$. This problem should be considered within a special study. 


\section{Conclusions}

We have derived the vortex-modified nonlinear Schrödinger equation using the method of multiple-scale expansions in the Lagrangian variables. The fluid vorticity $\Omega$ is specified as an arbitrary function of the Lagrangian coordinates, which is quadratic in the small parameter of the wave steepness. The calculations have been performed by introducing a complex coordinate of the fluid particle trajectory.

The nonlinear evolution equation for the wave packet in the form of the nonlinear Schrödinger equation has been derived as well. From the mathematical viewpoint, the novelty of this equation is related to the emergence of a new term proportional to the envelope amplitude and the variance of the coefficient of the nonlinear term. If the vorticity depends on the vertical Lagrangian coordinate only (Gouyon waves), this coefficient is constant. There are special cases when the coefficient of the nonlinear term equals zero and the resulting nonlinearity disappears. The Gerstner wave belongs to the latter case. Another effect revealed in the present study is the relation of the vorticity to the wave number shift in the carrier wave. This shift is constant for the modulated Gouyon wave. If the vorticity depends on both Lagrangian coordinates, the shift of the wave number is horizontally inhomogeneous. It is shown that the solution to the NLS equation for weakly rotational waves in the Eulerian variables may be obtained from the Lagrangian solution with an ordinary change in the horizontal coordinates.

Data availability. No data sets were used in this article.

Competing interests. The authors declare that they have no conflict of interest.

Acknowledgements. E. Pelinovsky appreciates the support obtained from the RNF under grant 16-17-00041. The authors wish to thank the editor, Roger Grimshaw, the reviewers for their very useful comments, and Nadezhda Krivatkina for providing English corrections.

Edited by: R. Grimshaw

Reviewed by: two anonymous referees

\section{References}

Abrashkin, A. A. and Yakubovich, E. I.: Vortex Dynamics in the Lagrangian Description, Fizmatlit, Moscow, 2006 (in Russian).

Abrashkin, A. A. and Zen'kovich, D. A.: Vortical stationary waves on shear flow, Izvestiya, Atmos. Ocean. Phys., 26, 35-45, 1990.

Akhmediev, N. N., Eleonskii, V. M., and Kulagin, N. E.: Generation of periodic trains of picosecond pulses in an optical fiber: exact solutions, J. Exp. Theor. Phys., 89, 1542-1551, 1985.

Baumstein, A. I.: Modulation of gravity waves with shear in water, Stud. Appl. Math., 100, 365-390, 1998.

Bennett, A.: Lagrangian Fluid Dynamics, Cambridge University Press, Cambridge, 2006.

Benney, D. J. and Maslowe, S. A.: The evolution in space and time of nonlinear waves in parallel shear flows, Stud. Appl. Math., 54, 181-205, 1975.

Benney, D. J. and Newell, A. C.: The propagation of nonlinear wave envelopes, J. Math. Phys., 46, 133-139, 1967.

Benney, D. J. and Roskes, G. J.: Wave instabilities, Stud. Appl. Math., 48, 377-385, 1969.

Colin, T., Dias, F., and Ghidaglia, J. M.: On rotational effects in the modulations of weakly nonlinear water waves over finite depth, Eur. J. Mech. B, 14, 775-793, 1995.

Davey, A.: The propagation of a weak nonlinear wave, J. Fluid Mech., 53, 769-781, 1972.

Dubreil-Jacotin, M. L.: Sur la détermination rigoureuse des ondes permanentes périodiques d'ampleur finie, J. Math. Pure. Appl., 13, 217-291, 1934.

Gouyon, R.: Contribution à la théorie des houles, Annales de la Faculté des Sciences de l'Université de Toulouse, 22, 1-55, 1958.

Grimshaw, R., Slunyaev, A., and Pelinovsky, E.: Generation of solitons and breathers in the extended Korteweg-de-Vries equation with positive cubic nonlinearity, Chaos, 20, 013102, https://doi.org/10.1063/1.3279480, 2010.

Hasimoto, H. and Ono, H.: Nonlinear modulation of gravity waves, J. Phys. Soc. Jpn., 33, 805-811, 1972.

Henderson, K. L., Peregrine, D. H., and Dold, J. W.: Unsteady water wave modulations: fully nonlinear solutions and comparison with the nonlinear Shrödinger equation, Wave Motion, 29, 341361, 1999.

Hjelmervik, K. B. and Trulsen, K.: Freak wave statistics on collinear currents, J. Fluid Mech., 637, 267-284, 2009.

Johnson, R. S.: On the modulation of water waves on shear flows, P. R. Soc. London, 347, 537-546, 1976.

Kuznetsov, E. A.: Solitons in a parametrically unstable plasma, Sov. Phys. Dokl., 22, 507-509, 1977.

Lamb, H.: Hydrodynamics, 6th Edn., Cambridge University Press, 1932.

Li, J. C., Hui, W. H., and Donelan, M. A.: Effects of velocity shear on the stability of surface deep water wave trains, in: Nonlinear Water Waves, edited by: Horikawa, K. and Maruo, H., Springer, Berlin, 213-220, 1987.

Ma, Y.-C.: The perturbed plane-wave solutions of the cubic Schrödinger equation, Stud. Appl. Math., 60, 43-58, 1979.

McIntyre, M. E.: On the "wave momentum" myth, J. Fluid Mech., 106, 331-347, 1981.

Oikawa, M., Chow, K., and Benney, D. J.: The propagation of nonlinear wave packets in a shear flow with a free surface, Stud. Appl. Math., 76, 69-92, 1987. 
Onorato, M., Proment, D., and Toffoli, A.: Triggering rogue waves in opposing currents, Phys. Rev. Lett., 107, 184502, https://doi.org/10.1103/PhysRevLett.107.184502, 2011.

Peregrine, D. H.: Water waves, nonlinear Schrödinger equations and their solutions, J. Australian Math. Soc. Ser. B, 25, 16-43, 1983.

Shrira, V. I. and Slunyaev, A. V.: Nonlinear dynamics of trapped waves on jet currents and rogue waves, Phys. Rev. E, 89, 041002, https://doi.org/10.1103/PhysRevE.89.041002, 2014.

Slunyaev, A. V.: Anomalous high sea waves: physical mechanisms and simulation, Doctoral (Habilitation) Thesis, Institute of Applied Physics of Russian Academy of Sciences, Nizhny Novgorod, Russia, 337 pp., 2016 (in Russian).
Sretensky, L. N.: Theory of wave motion in the fluid, Nauka, Moscow, 1977 (in Russian).

Stocker, J. R. and Peregrine, D. N.: The current-modified nonlinear Schrödinger equation, J. Fluid Mech., 399, 335-353, 1999.

Thomas, R., Kharif, C., and Manna, M.: A nonlinear Schrödinger equation for water waves on finite depth with constant vorticity, Phys. Fluids, 24, 127102, https://doi.org/10.1063/1.4768530, 2012.

Yuen, H. C. and Lake, B. M.: Nonlinear deep water waves: Theory and experiment, Phys. Fluids, 18, 956-960, 1975.

Zakharov, V. E.: Stability of periodic waves of finite amplitude on the surface of a deep fluid, J. Appl. Mech. Tech. Phys., 9, 190194, 1968. 